\title{
AV Graft Stenosis
}

National Cancer Institute

\section{Source}

National Cancer Institute. AV Graft Stenosis. NCI Thesaurus. Code C114762.

Narrowing of the lumen of an arteriovenous graft. 